\title{
Constant-Time Neural Decoders for Some BCH Codes
}

\author{
Yuen-Hsien Tseng and Ja-Ling Wu \\ Department of Computer Science and Information Engineering, National Taiwan Univ., Taipei, R.O.C.
}

Abstract - In this paper, high-order neural networks (HONN) are shown to decode some BCH codes in constant-time with very low hardware complexity.

\section{Introduction}

HONN is a direct extension of the linear perceptron: it uses a polynomial consisting of a set of product terms as its discriminant function. Because a product term is isomorphic to a parity function and a two-layer perceptron for the parity function has been shown in [1], HONN has a simple realization if it is considered as having a set of parity networks in the first-half layer, followed by a linear perceptron in the second-half layer.

The main problem in using high-order neural networks for a specific application is to decide a proper set of product terms. We apply genetic algorithms [2] to this structure-adaptation problem.

\section{Genetic Algorithms}

Genetic algorithms begin with a randomly generated population of individuals. Each individual is evaluated to show its fitness. High-fitness individuals receive high selection probability for reproduction. A number of genetic operators, such as mutation and crossover, are then applied to the selected individuals to produce a new population of the next generation. This process repeats until desired solutions emerge.

The performance of genetic algorithms has been analyzed and shown that better individuals are sampled and recombined to yield even better individuals [2]. We have carefully defined the fitness function and genetic operators to achieve this favorable performance.

\section{Neural Network Decoders}

To illustrate the applicability of this approach, a $\mathrm{BCH}(15,2)$ code, which has a minimum distance 10 and is thus four-error correctable and five-error detectable, is investigated. The parity matrix of the $\mathrm{BCH}(15,2)$ code is

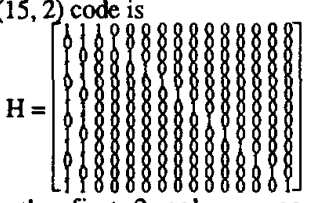

where the first 2 columns correspond to the 2 information bits and the last 13 columns correspond to the 13 parity bits such that the rows are the patterns indicating a parity bit and their constituent information bits. The obtained neural decoder is

$$
\begin{aligned}
g_{1}(X)= & x_{1}+x_{5}+x_{8}+x_{11}+x_{14}+x_{2} x_{3}+x_{6} x_{7}+ \\
& x_{4} x_{9}+x_{12} x_{13}+x_{10} x_{15} \\
g_{2}(X)= & x_{2}+x_{4}+x_{7}+x_{10}+x_{13}+x_{3} x_{8}+x_{1} x_{9}+ \\
& x_{6} x_{11}+x_{12} x_{14}+x_{5} x_{15}
\end{aligned}
$$

This solution can be verified by noticing that each variable in $g_{i}$ occurs exactly once and that each term in $\mathrm{g}_{\mathrm{i}}$ reduces to $\mathrm{x}_{\mathrm{i}}$ after replacing the parity bits with their constituent information bits. So any codeword patterns up to four bits in error (erroneous bit $x_{j}$ is represented as $-x_{j}$ ) lead to at most four negative terms $x_{i}$ and hence can be corrected due to majority (Summing $\mathbf{n}$ bipolar bits is tantamount to determining the majority of 1 or 1). The neural decoder can detect the existence of those five-bit errors for which case at least one of the discriminant polynomials is zero.

\section{Conclusion}

With the aid of the genetic algorithm, it is found that some t-error correctable codes can be decoded with $2 t+1$ terms for $2 t+2$ terms for $(t+1)$-error detection at the same time). Examples of these codes are listed in Table 1.

\section{References}

[1] D. E. Rumelhart, G. E. Hinton, and R .J. Williams, "Learning Internal Representations by Error Propagation" in Parallel Distributed Processing, D.E.Rumelhart and PDP research group, Cambridge, MA:MIT Press 1986.

[2] D. E. Goldberg, Genetic Algorithms in Search. Optimization, and Machine Learning, AddisonWesley, 1989.

\begin{tabular}{|c|c|c|c|l|c|}
\hline$n$ & $k$ & $d$ & ${ }^{\text {BCH }}$ & $\begin{array}{l}\text { Roots of } \\
\text { Gen.Poly. }\end{array}$ & $\begin{array}{c}\text { No. of } \\
\text { Tems }\end{array}$ \\
\hline 15 & 7 & 5 & 5 & 1,3 & 5 \\
\hline 15 & 6 & 6 & 6 & $0,1,3$ & 5 \\
\hline 15 & 4 & 8 & 8 & $0,1,3,5$ & 8 \\
\hline 15 & 3 & 5 & 5 & $1,3,7$ & 5 \\
\hline 15 & 2 & 10 & 10 & $0,1,3,7$ & 10 \\
\hline 21 & 11 & 6 & 6 & $0,1,3$ & 5 \\
\hline
\end{tabular}

Table 1. Some BCH codes and their corresponding number of terms required for decoding. 